\title{
Time-Weissenberg Number Superposition in 4:1 Planar Contraction Flow of a Viscoelastic Fluid
}

\author{
Ju Min KIM ${ }^{1}$, Changkwon CHung, Kyung Hyun $\mathrm{AHN}^{*}$, and Seung Jong LEE \\ School of Chemical and biological Engineering, Seoul National University, Seoul 151-744, Korea \\ ${ }^{1}$ Department of Chemical and Biological Engineering, Korea University, Seoul 136-701, Korea \\ (Received : March 9, 2005)
}

\begin{abstract}
Vortex enhancement mechanism in 4:1 planar contraction flow of a viscoelastic fluid has been investigated, where the Oldroyd-B model was considered as a constitutive equation. Finite element method was used based on the fractional step method for time marching and DEVSS/DG as a stabilizing scheme. The prediction of vortex dynamics agreed well with the experimental results. At first, a lip vortex was generated at the reentrant corner at low Weissenberg number $(W e)$. As We increases, the size of the salient corner vortex becomes reduced, while the lip vortex becomes more pronounced and finally takes over the corner vortex. The trajectory of a lip vortex at high We was found to follow the steady state positions of the lip vortex at lower $W e$, which means that the fluid has a memory and there exists a simple relationship between $W e$ and time. Accordingly, a simple universal behavior was found to dominate the vortex enhancement mechanism in the 4:1 planar contraction flow of the Oldroyd-B fluid.
\end{abstract}

Key Words: Time-We superposition / Intramolecular interaction / Lip vortex / Oldroyd-B / DEVSS-G/DG

\section{INTRODUCTION}

Contraction flow of a viscoelastic fluid occurs in many industrial applications, and has been studied for many years. The flow behavior is very complex due to a viscoelastic effect; a lip vortex is formed in the planar flows in addition to the corner vortex, and then it increases and finally takes over the corner vortex forming a big single vortex as the Weissenberg number $(W e=\lambda \dot{\gamma}$, where $\lambda$ is the relaxation time of a fluid and $\dot{\gamma}$ is the shear rate at downstream) increases. It is now regarded as a benchmark problem in the field of computational rheology, but the vortex growth mechanism is not fully understood yet. Numerical simulation of a viscoelastic fluid in such a complex geometry is a challenging problem. There exist at least two types of difficulties; one from the complex geometry and the other from the constitutive equation. The geometry has a corner singularity at the reentrant corner where the stresses are concentrating, and the viscoelastic constitutive equation (Oldroyd-B model in this case) has a hyperbolic character in the upper convected time derivative term in the model equation forming a set of first order hyperbolic partial differential equations. With these difficulties, it becomes numerically unstable and difficult to solve as the Weissenberg

* To whom correspondence should be addressed. ahnnet@snu.ac.kr number becomes larger. There are many approaches to solve this notorious high Weissenberg number problem. One stream is to split the governing equations into elliptic saddle point problems for velocity and pressure fields, and the hyperbolic equations for extra stresses. Explicitly elliptic momentum equation $(E E M E)^{1)}$, elastic viscous stress splitting $(\mathrm{EVSS})^{2}$, discrete elastic viscous stress splitting (DEVSS) ${ }^{3)}$ and its modification (DEVSS-G) $)^{4}$ are the examples of this stream, all of which are to stabilize the solution at high Weissenberg number by separating the elliptic and hyperbolic equations. The other stream is to reinforce the elliptic terms in the governing equations by adding artificial diffusion to reduce the contribution from the convective terms that deteriorate the stability. Streamline upwind/Petrov Galerkin (SUPG) ${ }^{5}$, streamlined upwind $(\mathrm{SU})^{6}$ and discontinuous Galerkin (DG) ${ }^{7}$ are the examples of the second stream. With the help of these techniques, it now becomes possible to solve high Weissenberg number problem though not yet perfect.

In this paper, 4:1 planar contraction flow of the Oldroyd-B fluid has been investigated. Time-dependent problem was solved using the finite element method. DEVSS-G/DG was used to stabilize the solution and the fractional step method was modified to solve the transient viscoelastic fluid flow. We will show the vortex enhancement process and will compare the transient solution at high $W e$ with the steady 
solutions at lower $W e$. Surprisingly, there exists a similarity in the path of the transient solution at high $W e$ and the steady state positions of the lip vortices at lower Weissenberg numbers. In addition, we will show that this complex flow behavior is dominated by a simple relationship that relates the time and $W e$. It will be named as time-We superposition. As all these observations are the evidence of a memory effect of the viscoelastic fluid, the origin of the memory effect will be discussed in terms of a kinetic theory which defines the constitutive equation. In the next section, simulation method will be briefly introduced. Then, simulation results on lip vortex enhancement will be provided, and our observation on the relationship between time and $W e$ will be provided followed by discussion.

\section{NUMERICAL METHOD}

The flow domain is shown in Fig. 1 with upstream length $20 L$ and downstream length $50 L$, where $L$ is the half width at downstream channel. Only half domain was considered due to symmetry. Time-dependent problem was solved using the finite element method. DEVSS-G/DG was used to stabilize the solution and the fractional step method (FSM $)^{8), 9)}$ was modified to solve the transient viscoelastic fluid flow. Two important dimensionless numbers governing this complex flow are the Reynolds number $(R e=\rho U L / \eta)$ and the Weissenberg number $(W e=\lambda U / L)$ where $\rho$ is the density, $U$ is the average velocity at downstream, $\eta$ is the solution viscosity, and $\lambda$ is the relaxation time of the fluid. Then the governing equations are (all the variables are dimensionless hereafter)

$$
\begin{aligned}
& R e\left(\frac{\partial \boldsymbol{u}}{\partial t}+\boldsymbol{u} \cdot \nabla \boldsymbol{u}\right)=-\nabla p+\beta \nabla \cdot\left(\nabla \boldsymbol{u}+(\nabla \boldsymbol{u})^{\boldsymbol{T}}\right)+\nabla \cdot \tau_{p} \\
& \nabla \cdot \boldsymbol{u}=0 \\
& \tau_{p}+W e \tau_{p}=(1-\beta)\left(\nabla \boldsymbol{u}+(\nabla \boldsymbol{u})^{T}\right)
\end{aligned}
$$

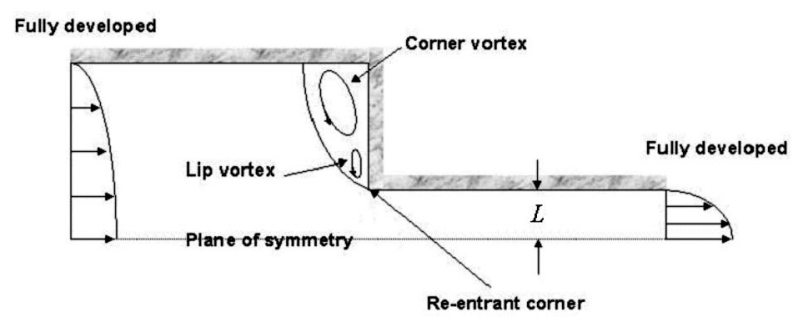

Fig. 1. Schematic diagram of the flow domain. where $p$ is the pressure, $\tau_{p}$ is the extra stress tensor, $\beta=n_{s} / n$ which represents the ratio of the solvent viscosity $\left(\eta_{s}\right)$ and the solution viscosity $(\eta)$ was set to $1 / 9, \boldsymbol{u}$ is the velocity vector, and $\stackrel{\nabla}{\tau}_{p}$ is the upper-convected derivative of the stress tensor $\tau_{p}$.

$$
\tau_{p}=\frac{\partial \tau_{p}}{\partial t}+\boldsymbol{u} \cdot \nabla \tau_{p}-(\nabla \boldsymbol{u})^{T} \cdot \tau_{p}-\tau_{p} \cdot(\nabla \boldsymbol{u})
$$

To stabilize the solution, DG was incorporated as following.

$$
\begin{aligned}
\left\langle\phi ; \boldsymbol{\tau}_{p}+\right. & \left.W e \boldsymbol{\tau}_{p}^{\nabla}-(1-\beta)\left(\nabla \mathbf{u}+(\nabla \mathbf{u})^{T}\right)\right\rangle \\
& -W e \sum_{e=1}^{N} \int_{\Gamma_{e}} \phi: \mathbf{u} \cdot \mathbf{n}\left(\boldsymbol{\tau}_{p}-\boldsymbol{\tau}_{p}^{e x t}\right) d \Gamma_{e}=0
\end{aligned}
$$

where $\left\langle A, B>\right.$ denotes $\int_{\Omega} A B d \Omega$ on $\Omega$ domain, $\boldsymbol{n}$ is a normal vector which has outward direction at the boundary of finite elements, and $\phi$ is the shape function. $\tau_{p}^{\text {ext }}$ takes the upstream additional stress value in the region of $\boldsymbol{u} \cdot \boldsymbol{n}<0$.

We adopted the fully implicit fractional step method ${ }^{10)}$, and carried out finite element formulation as following.

$$
\begin{aligned}
& \left\langle\boldsymbol{\phi} ; \operatorname{Re}\left(\frac{\hat{\boldsymbol{u}}-\boldsymbol{u}^{n}}{\Delta t}+\frac{1}{2}\left(\hat{\boldsymbol{u}} \cdot \nabla \hat{\boldsymbol{u}}+\boldsymbol{u}^{n} \cdot \nabla \boldsymbol{u}^{n}\right)\right)\right\rangle \\
& =-\left\langle\nabla \cdot \boldsymbol{\phi} ; p^{n}\right\rangle+\left\langle(\nabla \boldsymbol{\phi})^{T} ; \frac{1}{2}\left(\tau^{n+1}+\tau^{n}\right)+\right. \\
& -\left\langle(\nabla \boldsymbol{\phi})^{T} ;(1-\beta)\left(\frac{3}{2}\left(\boldsymbol{G}^{n}+\left(\boldsymbol{G}^{n}\right)^{T}\right)\right.\right. \\
& \left\langle\boldsymbol{\phi} ; \operatorname{Re} \frac{\boldsymbol{u}^{*}-\hat{\boldsymbol{u}}}{\Delta t}\right\rangle=\left\langle\nabla \cdot \boldsymbol{\phi} ; \frac{1}{2} p^{n}\right\rangle \\
& \left\langle\nabla \cdot \boldsymbol{\phi} ; \frac{1}{2} \nabla p^{n+1}\right\rangle=\left\langle\nabla \cdot \boldsymbol{\phi} ; \frac{\operatorname{Re}}{\Delta t} \boldsymbol{u}^{*}\right\rangle \\
& \left\langle\phi ; \operatorname{Re} \frac{\boldsymbol{u}^{n+1}-\boldsymbol{u}^{*}}{\Delta t}\right\rangle=-\left\langle\nabla \cdot \boldsymbol{\phi} ; \frac{1}{2} p^{n+1}\right\rangle .
\end{aligned}
$$$$
=-\left\langle\nabla \cdot \boldsymbol{\phi} ; p^{n}\right\rangle+\left\langle(\nabla \boldsymbol{\phi})^{T} ; \frac{1}{2}\left(\tau^{n+1}+\tau^{n}\right)+\frac{1}{2}\left(\nabla \hat{\boldsymbol{u}}+\nabla \boldsymbol{u}^{n}\right)\right\rangle
$$$$
-\left\langle(\nabla \boldsymbol{\phi})^{T} ;(1-\beta)\left(\frac{3}{2}\left(\boldsymbol{G}^{n}+\left(\boldsymbol{G}^{n}\right)^{T}\right)-\frac{1}{2}\left(\boldsymbol{G}^{n-1}+\left(\boldsymbol{G}^{n-1}\right)^{T}\right)\right)\right\rangle
$$

After calculating above steps, velocity gradient tensor $\mathbf{G}$ was projected.

$$
\left\langle\phi ; \boldsymbol{G}^{n+1}-\left(\nabla \boldsymbol{u}^{n+1}\right)^{T}\right\rangle=0
$$

Then, the solution procedure becomes as follows. First, the extra stress of the next step is calculated by solving Eq. (5). The 2nd order Adams-Bashforth method is employed for the extra stress and the solution is obtained element by element, 
which can be realized by the discontinuous Galerkin formulation. Then, the intermediate velocity is solved with $p$ being replaced by $p^{n}$ : this procedure is achieved by solving Eq. (6-1). However, the predicted velocity field does not satisfy the continuity so that it needs to be corrected. This is achieved by solving Eq. (6-3). Finally, the velocity gradient tensor is updated by Eq. (7). These procedures accomplish one time-step and continue until the steady solution is obtained.

In Fig. 1, no slip boundary condition was given at the solid wall, traction free boundary conditions were given at symmetry line as well as at downstream, a constant flow field as the parabolic profile was given at the inlet, and a pressure was specified as a reference value at the outlet. Initially, the velocity and extra stress fields were assumed to be quiescent in the flow domain.

As shown in Fig. 2, three different meshes were used in this work. More elements were concentrated near the singular point. The detailed information of meshes is given in Table I. Time increment was $O\left(10^{-3}\right)$ and the relative tolerance was less than $O\left(10^{-6}\right)$.
Table I Detailed information of mesh configuration

\begin{tabular}{crrrr} 
Name & Element & Node & \multicolumn{1}{c}{ DOF } & $\Delta \mathrm{l}_{\min } / \mathrm{L}$ \\
\hline M1 & 1,985 & 2,096 & 20,960 & 0.0128 \\
M2 & 9,437 & 9,682 & 96,820 & 0.0083 \\
M3 & 21,873 & 22,251 & 222,510 & 0.0050
\end{tabular}

\section{RESULTS AND DISCUSSION}

\subsection{Lip Vortex Enhancement}

The converged solution could be obtained up to $W e=9$ with M1 mesh. The streamlines are shown in Fig. 3. The lip vortex enhancement could be clearly observed as We increases. At $W e=1$, a small lip vortex appeared at the reentrant corner. As $W e$ increases, the size of the salient corner vortex becomes reduced, while the lip vortex becomes more stronger and finally takes over the corner vortex forming a big single vortex as is observed in some experiments. This means that the lip vortex enhancement is not a three dimensional phenomenon, and the Oldroyd-B model successfully predicts such a

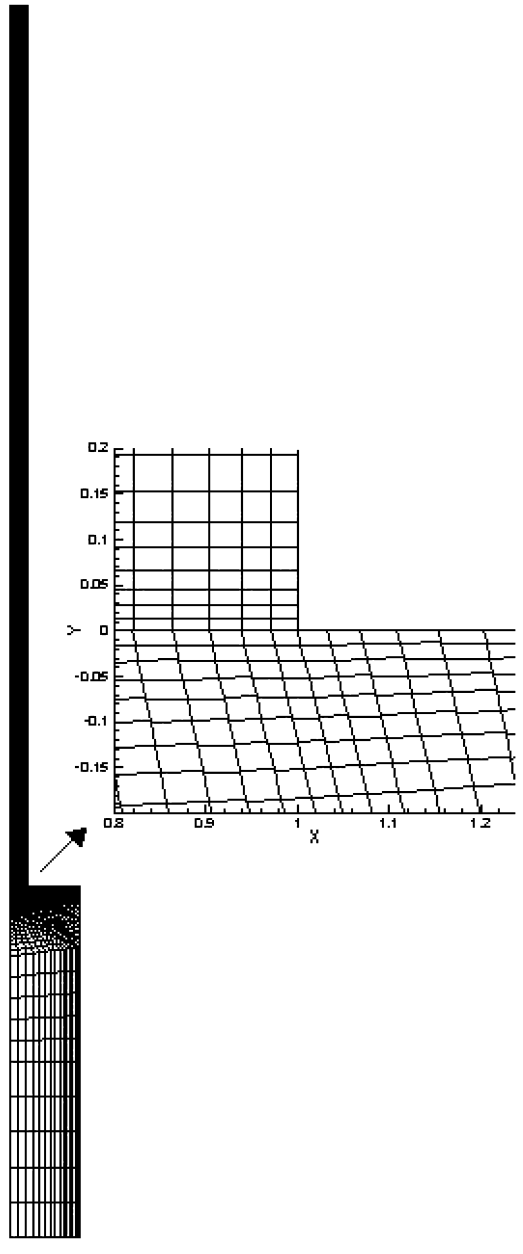

(a) M1
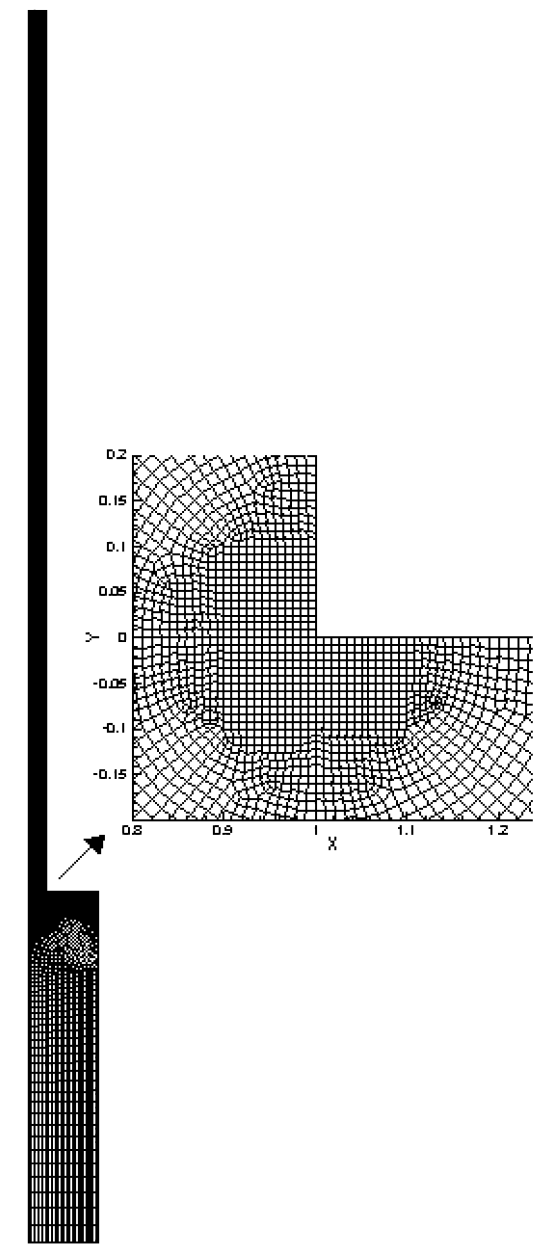

(b) $\mathrm{M} 2$

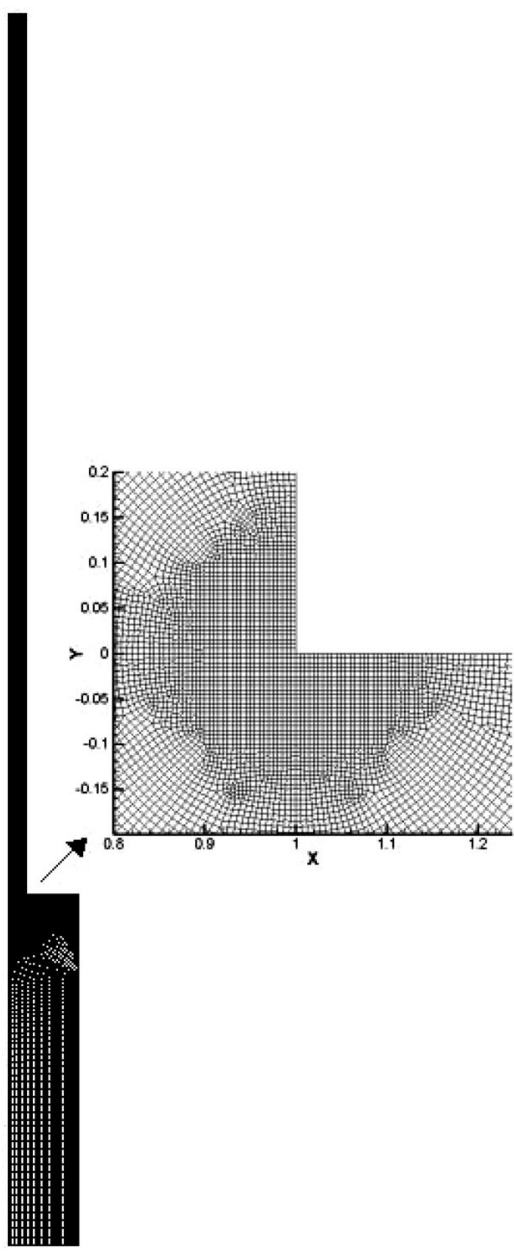

(c) M3

Fig. 2. Mesh configurations with full and zoomed view. 
complex flow behavior. In Fig. 4, we present the streamlines and velocity profiles along the centerline with increasing mesh density, which shows a good mesh convergence and confirms the robustness of the present numerical algorithm.

The co-existence of the lip and corner vortices, and the lip vortex enhancement were observed in the flow visualization experiments for 4:1 planar contraction flow with shearthinning fluids. ${ }^{11,{ }^{12)}}$ However, there has been no experimental evidence of lip vortex generation with the Boger fluid ${ }^{13)}$ in the 4:1 planar contraction flow. Only a small lip vortex was detected near the reentrant corner for high aspect ratio of 80:1 with the Boger fluid. ${ }^{11)}$ On the contrary, some previous researches demonstrated the growth of lip vortex using the constant shear viscosity model such as the Oldroyd-B or upper convected Maxwell model in planar 4:1 contraction flow geometry. ${ }^{14), 15)}$ Recent high-performance computation results confirm the existence and the growth of lip vortex for these models.9), 16), 17) Therefore, it can be deduced from previous

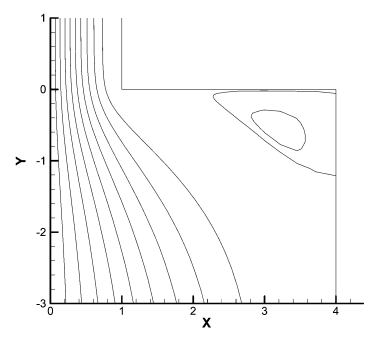

(a) $W e=0$

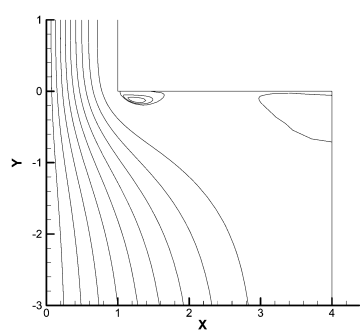

(c) $W e=3$

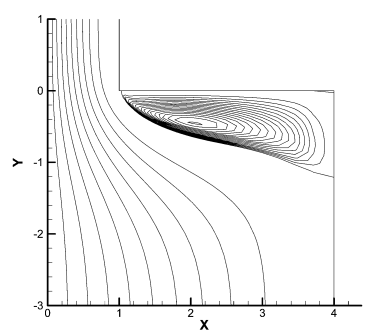

(e) $W e=7$

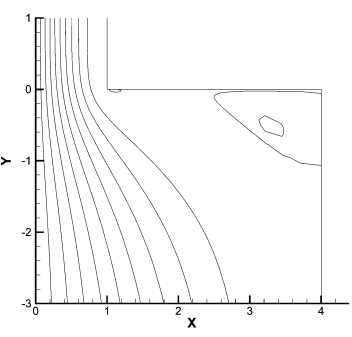

(b) $W e=1$

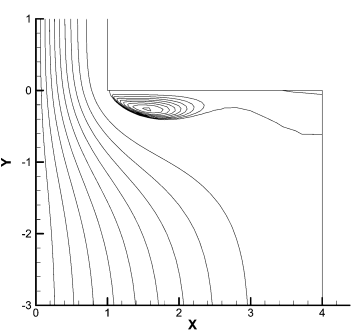

(d) $W e=5$

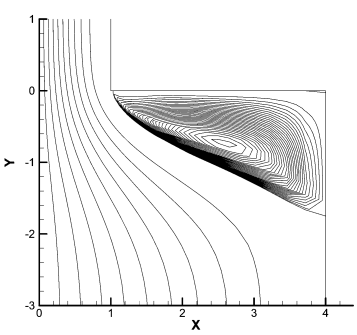

(f) $W e=9$

Fig. 3. Streamlines with increasing $W e\left(\Delta \psi=5 \times 10^{-4}\right)$.

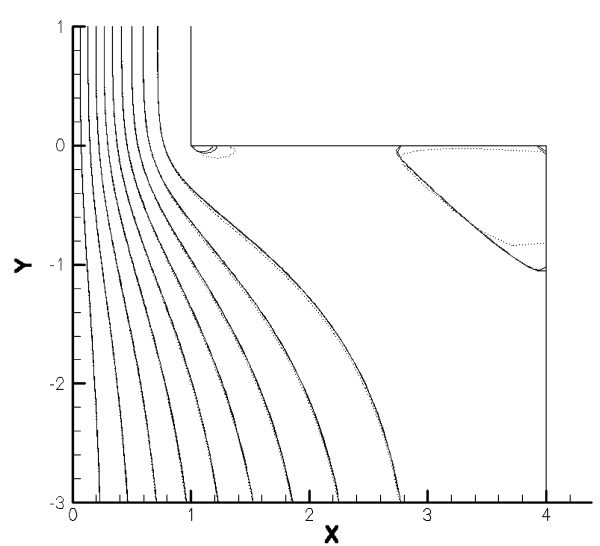

(a) Streamlines with increasing mesh density at $W e=2$.

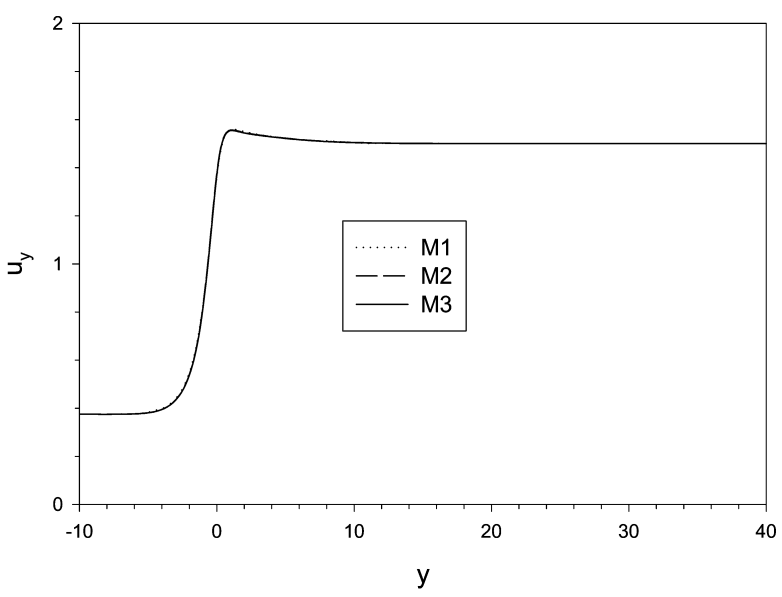

(b) Streamwise velocity profiles along the centerline at $W e=2$.

Fig. 4. Mesh dependence at $W e=2$ : dotted line (M1), dashed line (M2) and solid line (M3). 
at high We were compared to the vortex patterns of steady state solutions at lower We based on the position of the lip vortices. The trajectory of the lip vortex center is shown in Fig. 6. The vortex center was chosen as the node point with the minimum stream function, and a slight wriggling in the path arises from

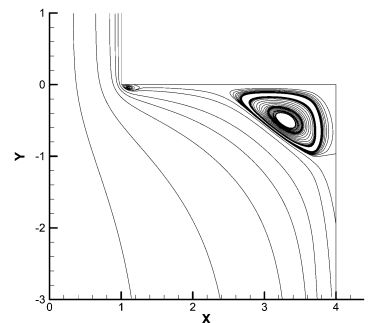

(a1) $\mathrm{t}=3.6(\mathrm{We}=9)$

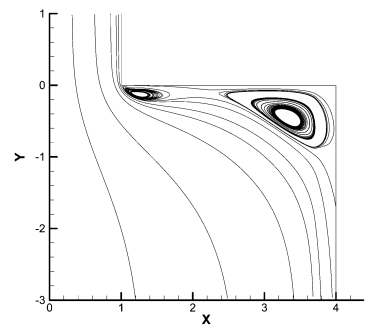

(b1) $\mathrm{t}=8.2(W e=9)$

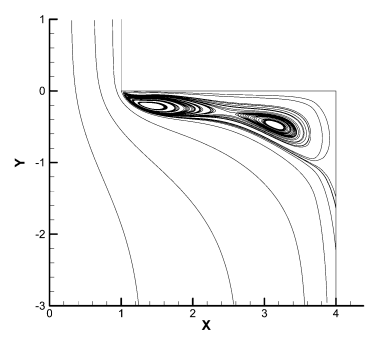

(c1) $\mathrm{t}=12.0(\mathrm{We}=9)$

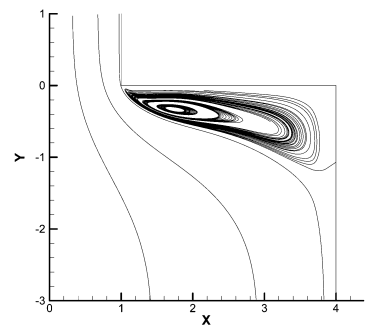

(d1) $\mathrm{t}=19.6($ We $=9)$

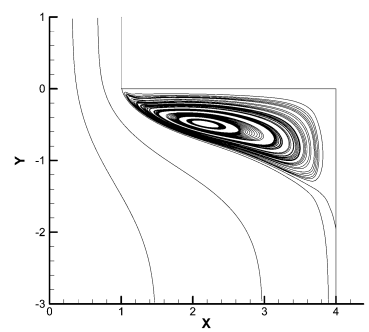

(e1) $\mathrm{t}=31.0(W e=9)$

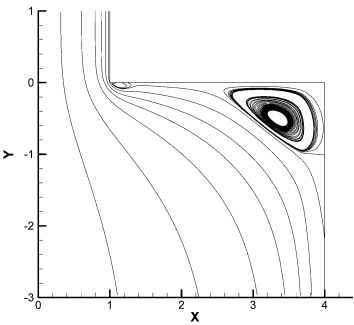

(a2) $W e=1.5$

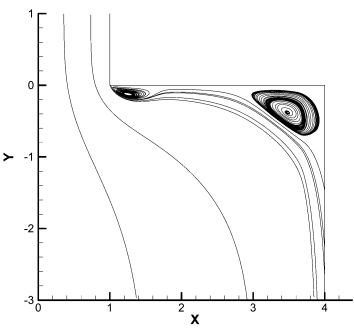

(b2) $W e=3.0$

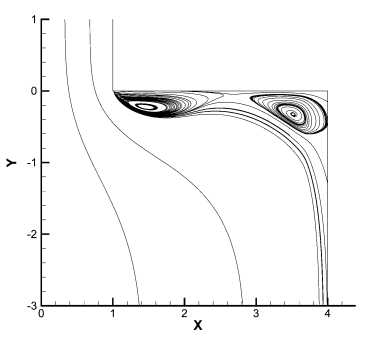

(c2) $W e=4.5$

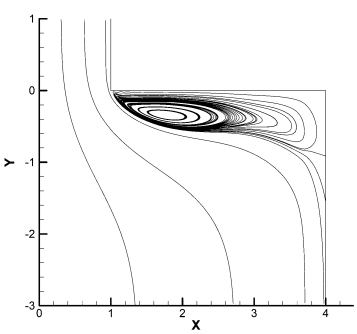

(d2) $W e=6.0$

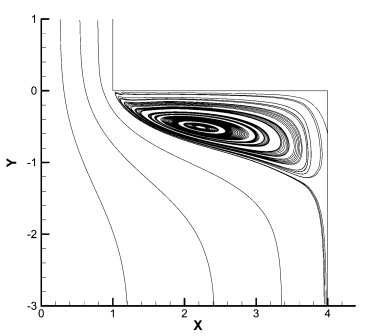

(e2) $W e=7.5$
Fig. 5. Comparison of transient solutions ( $W e=9$; (a1)-(e1)) with the steady solutions at lower $W e((\mathrm{a} 2)-(\mathrm{e} 2))$. the finite discretization. The solid line is the trajectory of the lip vortex center in the transient simulation at $W e=9$, and the symbols correspond to the steady state positions of the lip vortex at lower $W e(W e \leq 9)$. The trace of a lip vortex center at high We follows the steady state positions of the lip vortex centers at lower $W e$ as time elapses. When we used more refined mesh $\mathrm{M} 2$, the maximum attainable $W e$ was reduced, however, the superposition of the lip vortex could be confirmed as well. So, the superposition phenomenon seems to be independent of the mesh refinement.

Now we define the equivalent Weissenberg number $W e^{*}$, such that $W e^{*}$ is assigned to the transient state of high $W e(=9)$ flows when the transient position of the lip vortex center coincides with the steady position of the lip vortex center at lower $W e(W e \leq 9)$. Then the relationship between $W e^{*}$ and time in the transient simulation at $W e=9$ becomes very simple as shown in Fig. 7. The curve increases with time before the coalescence of two vortices, changes the slope at the coalescence and then saturates to a limiting $W e^{*}$. This relationship is almost universal regardless of $R e$ (though not shown here) and looks like a universal behavior underlying the vortex enhancement process. So, there exists a simple principle that dominates the vortex enhancement mechanism in this complex flow, and it may well be named as time-We superposition

The superposition could also be observed when we compare the intensities of vortices between the transient and steady solutions as shown in Fig. 8. The intensity of the lip vortex in the transient solutions almost overlaps with the ones of steady solutions. This peculiar coincidence as well as the coincidence of the position of the lip vortex center manifests a simple

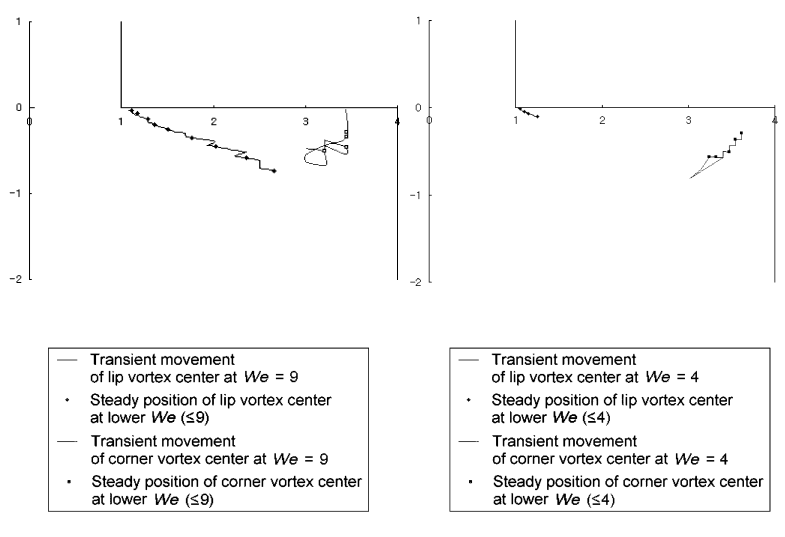

(a) trace of vortices (M1)

(b) trace of vortices (M2)

Fig. 6. Trajectory of centers of vortices. 
principle that governs the flow behavior even though the flow field looks very complex.

\subsection{Discussion}

From the viewpoint of a kinetic theory, the Oldroyd-B model pictures the dynamics of an infinitely dilute dumbbell immersed in a Newtonian medium. ${ }^{18)}$ The polymer chain is modeled as an extremely simple mechanical model; a dumbbell, two beads connected by a spring. Only the hydrodynamic drag force $F_{i}^{(h)}=-\zeta\left(\dot{r}_{i}-v_{i}\right)$ where $\zeta$ is the friction coefficient of a bead, $\dot{r}_{i}$ is the time derivative of the position vector of bead $i$ and $v_{i}$ is the imposed homogeneous velocity field at bead $i$, the equilibrium averaged Brownian force $F_{i}^{(b)}=-k T \partial \ln \Psi / \partial r_{i}$ where $\psi$ is the configuration-space distribution function, and the potential force $F_{i}^{(\varphi)}=-\partial \varphi / \partial r_{i}$ where $\varphi$ is the spring potential, are taken into account. It does not take intermolecular interaction, hydrodynamic interaction, excluded volume effect, or any nonlinear spring effect into account. Only the intramolecular interaction is taken into account as a form of Hookean spring potential, in which the detailed interaction is not described precisely but the force is defined by the Hookean spring constant multiplied by the distance between the beads. It is surprising how this simple dumbbell model can have a memory effect in such a complex flow behavior. This may be related to the relaxation time of the fluid or to the convected derivative terms in the constitutive equation (they always appear altogether). The Weissenberg number is defined by the relaxation time multiplied by shear rate. As we increase $W e$ with fixed flow rate in the simulation (we did it by fixing the boundary conditions the same), the increase of $W e$ is solely related to the increase of the relaxation time of the fluid, which is defined as $\lambda=\zeta / 4 H$ where $\zeta$ is the friction coefficient of a bead and $H$ is a spring constant. Friction coefficient $\zeta$ is defined as $\zeta=6 \pi \eta_{s} R$, where $\eta_{s}$ is the solvent viscosity and $R$ is the radius of a bead. However, when we increase $W e$, the size of the bead does not necessarily change and the friction coefficient remains the same, so the increase of We purely depends on the spring constant $H$, that is on the intramolecular interaction force. Therefore, from the view point of a kinetic theory, the memory effect observed in this complex nonlinear flow originates from the intramolecular interaction of a polymer chain. As the intramolecular interaction under flow environment represents the chain conformation, a single dumbbell responds to the environment in a simple unified manner. As there is no relaxation spectrum and there is no stochastic origin, it is not surprising to see a universal behavior even in the complex flow field. This will be the reason why we have a universal behavior in the complex flow behavior of the Oldroyd-B fluid. It is well known that the complex fluid has a memory and remembers the strain history. ${ }^{19), 20)}$ The stress at present time $t$ depends on the history of the stress for all past time $\left(-\infty<t^{\prime}<t\right)$. The memory effect in the linear regime is usually accounted for by the relaxation modulus $G(t)=G_{0} \exp (-t / \lambda)$, and the effect in the nonlinear regime is a little bit more complex but in more simple manner than expected as is observed in Fig. 5. Memory effect of polymer solution has been confirmed by experiments, and the universal behavior is often observed especially in the large scale motion. Our results add another evidence for the universal behavior of polymer dynamics, but this time in the complex flow field.

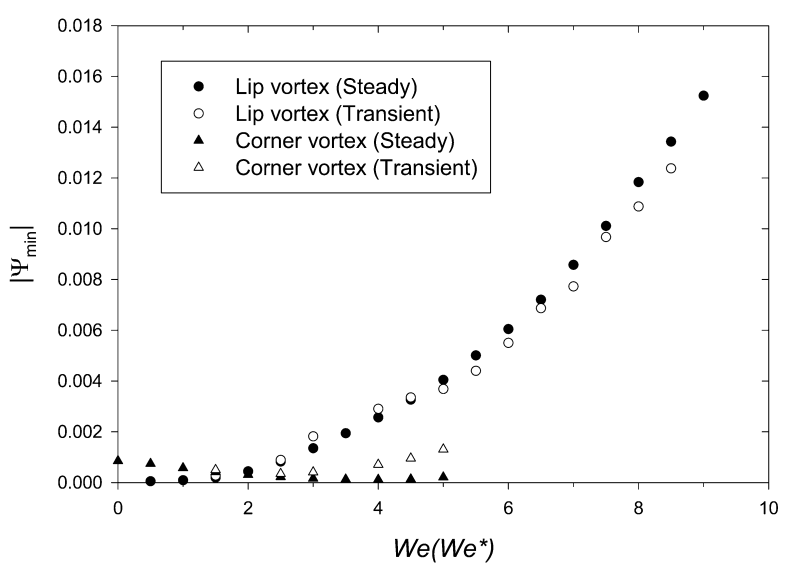

Fig. 8. Comparison of vortex intensities. $\left|\Psi_{\text {min }}\right|$ is the minimum stream function at each vortex center.

Fig. 7. $W e^{*}$ vs. time $(W e=9, R e=0.1)$. 


\section{CONCLUSION}

In summary, transient 4:1 planar contraction flow of the Oldroyd-B fluid was investigated by finite element simulation with FSM/DEVSS-G/DG. It was found that the transient position of a lip vortex at high $W e$ follows the steady state positions of the lip vortex at lower We. A universal behavior in the vortex enhancement mechanism was observed in this complex flow. The relationship between $W e$ and time may be called as time-We superposition. This interesting relationship was discussed in regard to the kinetic aspect of the constitutive equation, which simply postulates a Hookean dumbbell immersed in a Newtonian solvent with no hydrodynamic interaction or other intermolecular interactions except the intramolecular interaction depicted by a Hookean spring. The origin of this universal behavior was explained as a memory effect of the viscoelastic fluid in terms of the parameters in the kinetic theory.

\section{Acknowledgments}

This work was supported by the National Research Laboratory Fund (NRL 400-20030085) of the Ministry of Science and Technology in Korea. The authors also acknowledge the support from KISTI (Korea Institute of Science and Technology Information), and the use of the computing system of Supercomputing Center is greatly appreciated.

\section{REFERENCES}

1) King RC, Apelian MR, Armstrong RC, Brown RA, J NonNewtonian Fluid Mech, 29, 147 (1988).

2) Rajagopalan D, Armstrong RC, Brown RA, J Non-Newtonian Fluid Mech, 36, 159 (1990).
3) Guénette R, Fortin M, J Non-Newtonian Fluid Mech, 60, 27 (1995).

4) Liu AW, Bornside DE, Armstrong RC, Brown RA, J NonNewtonian Fluid Mech, 77, 153 (1998).

5) Brooks AN, Hughes TJR, Comput Methods Appl Mech Eng, 32, 109 (1982).

6) Marchal JM, Crochet MJ, J Non-Newtonian Fluid Mech, 26, 77 (1987).

7) Lesaint P, Raviart RA, "Mathematical Aspects of Finite Elements in Partial Differential Equations", (1974), Academic Press, New York.

8) Choi HG, Choi H, Yoo JY, Comput Methods Appl Mech Engrg, 143, 333 (1997).

9) Kim JM, Kim C, Kim JH, Chung C, Ahn KH, Lee SJ, J NonNewtonian Fluid Mech, accepted for publication (2005).

10) Choi H, Moin P, J Comput Phys, 113, 1 (1994).

11) Evans RE, Walters K, J Non-Newtonian Fluid Mech, 20, 11 (1986).

12) Evans RE, Walters K, J Non-Newtonian Fluid Mech, 32, 95 (1989).

13) Nigen S, Walters K, J Non-Newtonian Fluid Mech, 102, 343 (2002).

14) Oliveira PJ, Pinho FT, $J$ Non-Newtonian Fluid Mech, 88, 63 (1999).

15) Aboubacar M, Matallah H, Tamaddon-Jahromi HR, Webster MF, J Non-Newtonian Fluid Mech, 104, 125 (2002).

16) Alves MA, Oliveira PJ, Pinho FT, J Non-Newtonian Fluid Mech, 110, 45 (2003).

17) Edussuriya SS, Williams AJ, Bailey C, J Non-Newtonian Fluid Mech, 117, 47 (2004).

18) Bird RB, Curtiss CF, Armstrong RC, Hassager O, "Dynamics of Polymeric Liquids, vol. 2”, (1987), John Wiley \& Sons, New York.

19) Larson RG, "The Structure and Rheology of Complex Fluids", (1999), Oxford University Press, New York.

20) Trappe V, Weitz DA, Phys Rev Lett, 85, 449 (2000). 